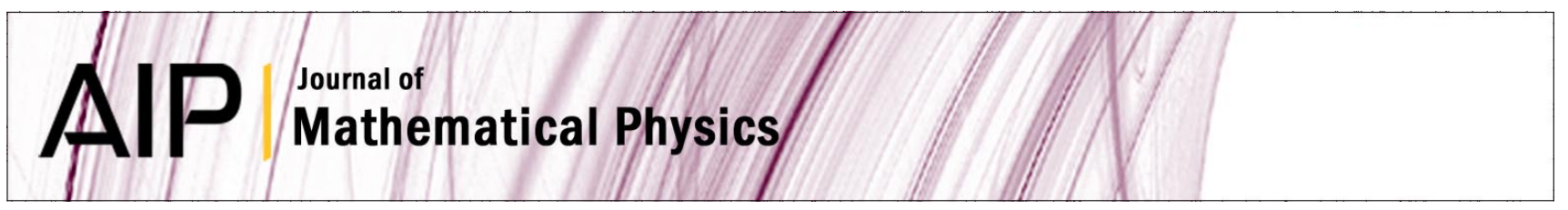

\title{
Description of the degree of nonuniqueness in inverse source problems
}

\author{
A. G. Ramm
}

Citation: J. Math. Phys. 25, 1791 (1984); doi: 10.1063/1.526356

View online: http://dx.doi.org/10.1063/1.526356

View Table of Contents: http://jmp.aip.org/resource/1/JMAPAQ/v25/i6

Published by the American Institute of Physics.

\section{Additional information on J. Math. Phys.}

Journal Homepage: http://jmp.aip.org/

Journal Information: http://jmp.aip.org/about/about_the_journal

Top downloads: http://jmp.aip.org/features/most_downloaded

Information for Authors: http://jmp.aip.org/authors

\section{ADVERTISEMENT}

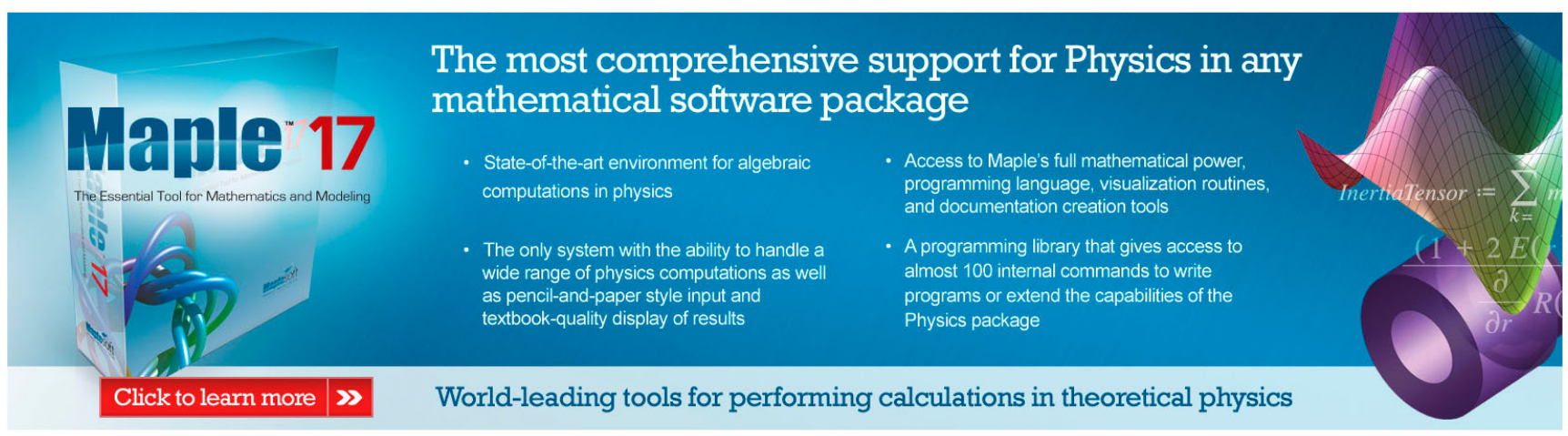




\title{
Description of the degree of nonuniqueness in inverse source problems
}

\author{
A. G. Ramm \\ Mathematics Department, Kansas State University, Manhattan, Kansas 66506
}

(Received 5 April 1983; accepted for publication 14 October 1983)

For time-independent and time-dependent inverse source problems the degree of nonuniqueness of solutions is characterized.

PACS numbers: 03.50.De, 03.65.Nk, 03.80. $+\mathrm{r}$

\section{INTRODUCTION}

1. Recently a number of authors discussed the inverse source problems ${ }^{1-5}$ (see also the bibliography in Refs. 1 and 5). The problem under discussion is as follows. Let

$$
\left(\nabla^{2}+k^{2}\right) u=h, \quad x \in \mathbb{R}^{3} ; \quad h=0, \quad|x| \geqslant a .
$$

Suppose the Cauchy data is given for $u$ near infinity:

$$
\left.u\right|_{|x|=R}=u_{0},\left.\quad \frac{\partial u}{\partial r}\right|_{|x|=R}=u_{1}, \quad R>1 .
$$

Can one determine $h$ from the data (2)?

Most of the authors ask a slightly different but equivalent question. Namely, can one determine $h$ from the radiation pattern $f(\theta, \phi, k)$, which is defined by the formula

$$
\begin{aligned}
& f(\theta, \phi, k)=\lim _{r \rightarrow+\infty}\left(\frac{e^{i k r}}{4 \pi r}\right)^{-1} u(x, k), \\
& r=|x|, \quad x=(r, \theta, \phi)
\end{aligned}
$$

where $u$ is the (unique) solution to (1) satisfying the radiation condition? The equivalence of the data (3) and (4) comes from the fact that the asympotics of $u$ at infinity can be differentiated. On a more formal level, the Cauchy data (3) defines the solution of the homogeneous equation (1) uniquely in the domain $|x| \geqslant a$, and the radiation pattern (4) does the same (because of the Rellich's uniqueness theorem, see, e.g., Ref. 6). The problem (3) is the time-independent inverse source problem.

2. This problem for Maxwell's equations was discussed in detail in Ref. 7 (see also Ref. 8, pp. 208-211). The problem for Maxwell equations has some features which the scalar problem (1)-(4) does not have. In particular, the radiation patterns for Maxwell's equations is a two-component vector field defined on the unit sphere $S^{2} \subset \mathbb{R}^{3}$ and tangent to this sphere (it is two-component in the spherical coordinates: $f_{r}$ $=0$ ). On the other hand, the source $j(x)$ in Maxwell's equations:

$$
\nabla \times E=i k H, \quad \operatorname{rot} H=-i k E+j, \epsilon=\mu=1,
$$

is a three-component vector. The corresponding radiation pattern is

$$
f=i k\left(I_{\phi} a_{\phi}+I_{\theta} a_{\theta}\right) \text {, }
$$

where $a_{r}, a_{\theta}, a_{\phi}$ are the unit vectors of the spherical coordinates at the point $x=(r, \theta, \phi), I=\left(I_{r}, I_{\theta}, I_{\phi}\right)$,

$$
I=\int \exp \{-i(\mathbf{k}, y)\} j(y) d y, \quad \mathbf{k}=(k, \theta, \phi), \quad \int \equiv \int_{\mathbf{R}^{3}} .
$$

It is clear from (5) that the radiation pattern $f(k, \theta, \phi)$ given for a fixed $k=k_{0}$ as a vector field on the unit sphere does not determine $I$ uniquely, and therefore does not determine the sources $j$ uniquely. The degree of nonuniqueness can be described as follows: in order to determine the sources uniquely one should specify: (1) one scalar function $I_{r}\left(k_{0}, \theta, \phi\right)$, and (2) three functions $f_{\theta}(k, \theta, \phi), f_{\phi}(k, \theta, \phi), I_{r}(k, \theta, \phi), 0<k<\infty$, which are equal to the functions $f_{\theta}\left(k_{0}, \theta, \phi\right), f_{\phi}\left(k_{0}, \theta, \phi\right)$, $I_{r}\left(k_{0}, \theta, \phi\right)$ at $k=k_{0}$. Here we did not impose the important requirement that the sources have compact support. If this requirement is imposed then the functions $I_{r}(k, \theta, \phi)$, $f_{\theta}(k, \theta, \phi), f_{\phi}(k, \theta, \phi)$ should satisfy the condition that the vector function $I=I_{r} a_{r}+(1 / i k) f_{\phi} a_{\phi}+(1 / i k) f_{\theta} a_{\theta}$ be an entire function of $\mathbf{k}$ of exponential type, i.e., $|I| \leqslant c_{1} \exp \left(c_{2} k\right)$ for some constants $c_{1}, c_{2}>0$. This requirement comes from the Paley-Wiener theorem which says that a function $I(\mathbf{k})$ is a Fourier transform of a function $j$ with compact support iff $I$ is an entire function of exponential type. In addition, $j \in L^{2}$ if $I \in L^{2}\left(\mathbb{R}^{3}\right)$. This analysis, a computational scheme for finding $j$ from the radiation pattern, and some examples are given in Ref. 7.

One should have in mind that in some concrete inverse source problems (e.g., synthesis of linear or spherical antennas) the uniqueness of the solution holds because of some special assumptions about the sources. There is an extensive literature on this subject (see, e.g., Ref. 9). In order to explain how the uniqueness holds, consider the linear antenna synthesis problem. The sources are currents along the line segment $-l<z<l$. The currents are defined by one scalar function $j(z)$ in this case. The radiation pattern is proportional to $\int_{-1}^{l} \exp \left(i k_{0} z \cos \theta\right) j(z) d z=f(\theta), 0 \leqslant \theta \leqslant \pi$. This can be written as $\delta_{-l}^{l} \exp (i \lambda z) j(z) d z=f(\lambda),-k_{0} \leqslant \lambda \leqslant k_{0}$, $\lambda=k_{0} \cos \theta$. The last equation clearly has no more than one solution. It is solvable iff $f(\lambda)$ is an entire function of exponential type $\leqslant l$ of $\lambda$.

3. In the scalar case one has

$$
f=-\frac{1}{4 \pi} \int \exp \{-i(\mathbf{k}, \boldsymbol{y})\} h(y) d y .
$$

Thus, the knowledge of $f\left(k_{0}, \theta, \phi\right)$ does not determine the sources $h(y)$ uniquely. The degree of nonuniqueness can be described as follows: given the radiation pattern at $k=k_{0}$ one can fix a (arbitrary continuous in $k$ ) function $f(k, \theta, \phi)$ for $0<k<\infty$ such that $\left.f(k, \theta, \phi)\right|_{k=k_{0}}=f\left(k_{0}, \theta, \phi\right)$. These data determine the Fourier transform of the sources and there- 
fore the sources $h(y)$ uniquely. In this argument we did not impose any a priori conditions on the sources. If one assumes (as is natural) that $h \in L^{2}$ and $h=0$ for $|x| \geqslant a$, then the radiation pattern is an entire function of $\mathbf{k}$ of exponential type and the corresponding extension $f(k, \theta, \phi)$ should satisfy this necessary requirement. This requirement is also sufficient for $h \in L^{2}$ and $h=0,|x| \geqslant a$ if $f \in L^{2}$. This is a complete description of the degree of nonuniqueness in the scalar problem of finding the sources from the radiation pattern given at a fixed frequency.

4. The reason why the problem (3) is discussed here is that the similar problem arises in time-dependent cases. Consider, for example, the following time-dependent inverse source problem. Let

$$
\begin{aligned}
& u_{t t}-\Delta u=f(x, t), \quad-\infty<t<\infty, \quad x \in \mathbb{R}^{3}, \\
& f=0 \text { of } t \notin(0, T) .
\end{aligned}
$$

Suppose that

$$
\begin{array}{ll}
u(0, x)=u_{0}(x), & u_{t}(0, x)=u_{1}(x), \\
u(T, x)=v_{0}(x), & u_{t}(T, x)=v_{1}(x) .
\end{array}
$$

Can one find the sources $f(x, t)$ from the data (7), (8)?

Because of the uniqueness of the Cauchy problem for Eq. (6) the data (7), (8) determine the solution of (7) for $t<0(t>T)$ uniquely. In Sec. II the description of the degree of nonuniqueness of the solution to problems (3) and (9) are given.

\section{DESCRIPTION OF THE DEGREE OF NONUNIQUENESS IN THE INVERSE SOURCE PROBLEMS}

1. Consider first problem (9). Taking the Fourier transform in $x$, one obtains

$$
\begin{aligned}
& \ddot{w}+k^{2} w=g(t, \mathbf{k}), \\
& w=\frac{1}{(2 \pi)^{3}} \int u(x, t) \exp \{-i(\mathbf{k}, x)\} d x \equiv \tilde{u}, \quad g \equiv \tilde{f}, \\
& w(0, \mathbf{k})=w_{0} \equiv \tilde{u}_{0}, \quad \dot{w}(0, \mathbf{k})=w_{1} \equiv \tilde{u}_{1}, \quad \dot{w}=\frac{d w}{d t}, \\
& w(T, \mathbf{k})=p_{0} \equiv \tilde{v}_{0}, \quad \dot{w}(T, \mathbf{k})=p_{1} \equiv \tilde{v}_{1} .
\end{aligned}
$$

Here $\mathbf{k}=\left(k_{1}, k_{2}, k_{3}\right), k^{2}=k_{1}^{2}+k_{2}^{2}+k_{3}^{2}, k=|\mathbf{k}|$. The problem (10), (11) can be solved explicitly,

$$
\begin{aligned}
w= & \int_{0}^{t} \frac{\sin \{k(t-s)\}}{k} g(s, \mathbf{k}) d s \\
& +w_{0} \cos (k t)+w_{1} \frac{\sin k t}{k} .
\end{aligned}
$$

From (13) and (12) one obtains

$$
\begin{aligned}
p_{0}= & \int_{0}^{T} \frac{\sin \{k(T-s)\}}{k} g d s \\
& +w_{0} \cos (k T)+w_{1} \frac{\sin (k T)}{k} \\
p_{1}= & \int_{0}^{T} \cos \{k(T-s)\} g d s-k w_{0} \sin (k T)
\end{aligned}
$$

$$
+w_{1} \cos k T \text {. }
$$

This can be written as

$$
\begin{gathered}
\frac{\sin (k T)}{k} \int_{0}^{T} \cos (k s) g d s-\cos (k T) \\
\times \int_{0}^{T} \sin (k s) g d s=b_{0}(k), \\
\frac{\cos (k T)}{k} \int_{0}^{T} \cos (k s) g d s+\sin (k T) \\
\times \int_{0}^{T} \sin (k s) g d s=b_{1}(\mathbf{k}),
\end{gathered}
$$

where $b_{0}, b_{1}$ are known explicitly:

$$
\begin{aligned}
& b_{0}=p_{0}-w_{0} \cos (k T)-\left(w_{1} / k\right) \sin k T, \\
& b_{1}=p_{1}+k \omega_{0} \sin (k T)-w_{1} \cos (k T) .
\end{aligned}
$$

The determinant of the system $(15)$ is $1 / k>0$. Thus, one can uniquely find from (15) the two integrals

$$
\begin{aligned}
& \int_{0}^{T} \cos (k s) g(s, \mathbf{k}) d s=\phi_{1}(\mathbf{k}), \\
& \int_{0}^{T} \sin (k s) g(s, \mathbf{k}) d s=\phi_{2}(\mathbf{k}),
\end{aligned}
$$

where $\phi_{1}(\mathbf{k})$ and $\phi_{2}(\mathbf{k})$ are given explicitly.

It is now possible to describe the degree of nonuniqueness of the solution to the inverse source problem (9). Namely, the data (11), (12) determine $\phi_{1}$ and $\phi_{2}$ in (17). Equations (17) determine $g$, the Fourier transform of the sources, of the form

$$
g=c_{1}(\mathbf{k}) \cos (k s)+c_{2}(\mathbf{k}) \sin (k s)+g_{1}(s, \mathbf{k}),
$$

where $c_{1}(\mathbf{k})$ and $c_{2}(\mathbf{k})$ are uniquely determined by $\phi_{1}$ and $\phi_{2}$, while $g_{1}(s, k)$ is an arbitrary function orthogonal to $\cos (k s)$ and $\sin (k s)$ in $L^{2}([0, T])$ :

$$
\begin{aligned}
\int_{0}^{T} \sin (k s) g_{1}(s, \mathbf{k}) d s \\
\quad=\int_{0}^{T} \cos (k s) g_{1}(s, \mathbf{k}) d s=0 \quad \forall k .
\end{aligned}
$$

This is a complete description of the degree of nonuniqueness of the solution to problem (9) in the case when no conditions are imposed on the sources $f(x, t)$ except the last condition (10) and, say, the mild requirement like $f(x, t) \in L^{2}\left(\mathbb{R}^{3}\right)$ for any $t$ and is of class $C^{2}$ in time. If one requires additionally that $f(x, t)=0$ for $|x| \geqslant a$ (the sources have compact support), then $g_{1}$ should satisfy not only Eqs. (19), but also ensure that the function $g$ defined by formula (18) be an entire function of exponential type in the variables $\mathbf{k}=\left(k_{1}, k_{2}, k_{3}\right)$. Since $g$ determines the sources $f$ uniquely, one can say that the solution of problem (9) is defined nonuniquely up to an additive term orthogonal to a two-dimensional subspace. The method of this section can be applied without any changes to the abstract time-dependent inverse source problem. This problem is similar to the problem (6)-(9), but Eq. (6) is of the form $u_{t r}+A u=f(t),-\infty<t<\infty$, where $A>0$ is a self-adjoint operator on a Hilbert space $h, f(t) \in H$. One assumes that the eigenfunction expansion theorem for $A$ is known and the eigenfunctions satisfy the equation $A \phi=k^{2} \phi$, and obtains 
the same equations (10)-(12) in which $w$ is the coefficient of the expansion of $u$ in the eigenfunctions $\phi$ (analog of the Fourier transform). The rest of the argument is unchanged.

2. Let us consider problem (1)-(3). The Cauchy data (2) determines uniquely the solution to Eq. (1) in the domain $|x| \geqslant a$ in which (1) is homogeneous. Let

$$
\left.u\right|_{r=a}=\psi_{0},\left.\frac{\partial u}{\partial r}\right|_{r=a}=\psi_{1},
$$

where $\psi_{0}$ and $\psi_{1}$ are uniquely determined by $u_{0}$ and $u_{1}$. (Of course, as is well known, the data $u_{0}$ and $u_{1}$ for an elliptic equation cannot be given arbitrarily.) One can also find $\psi_{0}$ and $\psi_{1}$ from the knowledge of the radiation pattern. Let $G(x, y)$ be the Green's function $\left(\nabla^{2}+k^{2}\right) G=-\delta(x-y)$, $|x| \leqslant a,\left.G\right|_{|x|=a}=0$. Then, the solution of the problem

$$
\left(\nabla^{2}+k^{2}\right) u=h, \quad|x| \leqslant a,\left.\quad u\right|_{|x|=a}=\psi_{0}
$$

can be found explicitly provided that $k^{2}$ is not an eigenvalue of the Dirichlet Laplacian in the domain $|x| \leqslant a$. Namely,

$$
u=\int_{\mathscr{S}} G(x, y) h d y+\int_{\Gamma} \frac{\partial G(x, s)}{\partial N} \psi_{0} d s, \quad x \in \mathscr{D},
$$

where $\mathscr{D}=\{x:|x| \leqslant a\}, \Gamma=\{x:|x|=a\}, N=N_{s}$ is the outer unit normal to $\Gamma$ at the point $s$. From (22) and the second condition (20) one obtains an equation for $h$. Since the problem is linear and one is interested in the description of nonuniqueness, one can take $\psi_{0}=\psi_{1}=0$. In this case the equation for $h$ which follows from (20) and (22) is of the form

$$
0=\int_{\mathscr{Z}} \frac{\partial G(s, y)}{\partial N} h d y, \quad s \in \Gamma
$$

To describe the degree of nonuniqueness of the solution to problem (3) one should describe the set of solutions to Eq. (23). If $\psi_{0}=0$ and $\psi_{1}=0$ then $u \equiv 0$ in $|x| \geqslant a$. Therefore one describes the nonradiating sources. This question was often discussed in the physical literature. ${ }^{5}$ The following is a description of the set of all solutions of Eq. (23). Let us multiply (23) by a smooth function $\phi(s)$ and integrate over $\Gamma$ to obtain

$$
0=\int_{\mathscr{Q}} d y h(y) F(y)
$$

where $F(y)=\int_{\Gamma}(\partial G(s, y) / \partial N) \phi(s) d s$ is the solution of the Dirichlet problem,

$$
\begin{aligned}
& \left(\nabla^{2}+k^{2}\right) F=0 \text { in } \mathscr{D}, \\
& \left.F\right|_{\Gamma}=-\phi .
\end{aligned}
$$

Therefore $F$ runs through the set of functions satisfying the homogeneous Helmholtz's equation in $\mathscr{D}$. Equations (23) and (24) are equivalent. Thus one obtains the following statement. The set of all solutions of (23) (i.e., the set of all nonradiating sources) is precisely the set of functions orthogonal to all of the solutions (in $L^{2}$ say) of Eq. (25). For example, if $\mathscr{D}$ is a ball of radius $a$ with center at the origin, then a basis in the set of solutions of Eq. (25) forms the functions $r^{-1 / 2} J_{n+1 / 2}(k r) Y_{n m}(\theta, \phi)$, where $J_{n}$ is the Bessel function and $Y_{n m}$ are the spherical harmonics.

Remark: It seems that the first paper on antenna synthesis theory was published in 1937 by my father. ${ }^{10}$

'A. Devaney and G. Sherman, "Nonuniqueness in inverse source and scattering problems,” IEEE Trans. Antennas Propag. AP-30, 1034-1037 (1982).

${ }^{2}$ A. Devaney, J. Math Phys. 19, 1526-1531 (1978); 20, 1687-1691 (1979).

${ }^{3}$ N. Bleistein and J. Cohen, J. Math. Phys. 18, 194-201 (1977).

${ }^{4}$ N. Bojarski, J. Math. Phys. 22, 1647-1650 (1981).

${ }^{5}$ Inverse Source Problems in Optics, edited by H. Bates (Springer-Verlag, New York, 1978.

${ }^{6}$ A. G. Ramm, J. Math. Phys. 22, 275-276 (1981).

${ }^{7}$ A. G. Ramm, On Antenna Synthesis Theory, Antennas, No. 5 (Izdat Svjaz, Moscow, 1969), pp. 35-46 (Russian).

${ }^{8}$ A. G. Ramm, Theory and Applications of Some New Classes of Integral Equations (Springer-Verlag, New York, 1980).

${ }^{9} \mathrm{~B}$. Minkovich and V. Jakovlev, Antenna Synthesis Theory (Soviet Radio, Moscow, 1967) (Russian).

${ }^{10} \mathrm{G}$. S. Ramm, "On antifeding antennas," in Collection of Scientific Papers on Electrocommunication (Leningrad Institute of Electrical Engineering and Communication (LEEC), Leningrad, 1937), Vol. 3, p. 19. Also G. S Ramm, Ph.D. Thesis "Antifeding antennas," LEEC 1937. 